\title{
Correction to: Surveillance of Modern Motherhood
}

\section{Correction to:}

H. Simmons, Surveillance of Modern Motherhood, https://doi.org/10.1007/978-3-030-45363-3

The original version of the book was inadvertently published with incorrect abstracts, which have now been corrected. 